\title{
Bispectral index decreased to zero for a patient undergoing orthotopic liver transplantation
}

\author{
Yoon Ji Choi ${ }^{1}$, Eun Jung $\mathrm{Cho}^{2}$, Seung Zhoo Yoon ${ }^{2}$, Hye Won Lee ${ }^{2}$, and Hae Ja Lim ${ }^{2}$ \\ Department of Anesthesiology and Pain Medicine, ${ }^{1}$ Seoul Paik Hospital, College of Medicine, Inje University, ${ }^{2}$ College of Medicine, \\ Korea University, Seoul, Korea
}

A 56-year-old man (height, $165 \mathrm{~cm}$; weight, $75 \mathrm{~kg}$ ) suspected of having herbal-related fulminant hepatitis was admitted for emergency orthotopic liver transplantation (LT). His medical history was unremarkable, except for hypertension. Upon admission to our hospital, the patient was alert with a Glasgow Coma Scale (GCS) score of $15(4 / 5 / 6)$ that gradually decreased to $10(3 / 2 / 5)$ by $28 \mathrm{~h}$ after admission. After transfer to the intensive care unit (ICU), intubation was performed and a propofol infusion was started at $4 \mathrm{mg} / \mathrm{kg} / \mathrm{h}$. The patient's vital signs were stable, and mechanical ventilatory support was performed with air in $\mathrm{O}_{2}\left(\mathrm{FiO}_{2}\right.$ 0.5). Before surgery, the patient's Model for EndStage Liver Disease score was 21, and the Child-Pugh score was class C. The patient's pupils were $3 \mathrm{~mm} / 3 \mathrm{~mm}$ in diameter (left/ right), and brain computed tomography (CT) and magnetic resonance imaging (MRI) revealed no remarkable findings.

Upon arrival to the operating room, the right radial artery was cannulated and arterial blood pressure, electrocardiography, heart rate, pulse oxygen saturation, end-tidal $\mathrm{CO}_{2}$ concentration, and bispectral index (BIS) were monitored continuously. The preoperative BIS value was 43 . Anesthesia was induced and maintained with a $4-6 \mathrm{vol} \%$ of desflurane and a $5-15 \mathrm{ng} / \mathrm{ml}$ of target effect site-controlled remifentanil infusion. Mechanical ventilatory support was maintained with air in $\mathrm{O}_{2}\left(\mathrm{FiO}_{2}\right.$ 0.5). A Swan-Ganz catheter was inserted into the right internal jugular vein to monitor cardiac output and pulmonary arterial pressure. Prior to the skin incision, the patient's vital signs were stable, and his BIS value was 14 .

Approximately $1 \mathrm{~h}$ after the skin incision, the patient's BIS values decreased rapidly to zero. The suppression ratio was 100 , and there was an absence of electric activity on electroencephalography. The patient's pupils remained $3 \mathrm{~mm} / 3 \mathrm{~mm}$ (left/right) in diameter. His vital signs were stable, and his body temperature was $35.2^{\circ} \mathrm{C}$. The BIS sensors were checked for proper placement. An arterial blood gas analysis was performed to evaluate for metabolic disturbances, but the results did not show any abnormal findings.

During surgery, the patient's vital signs were generally stable (Table 1). We tried to maintain the mean arterial pressure at $>60 \mathrm{mmHg}$ and body temperature at $34-35^{\circ} \mathrm{C}$. Despite efforts to increase the BIS level, the patient's BIS values remained at zero until the end of the surgery for a total duration of $10 \mathrm{~h} 50$ min. The surgical procedures were completed uneventfully with a total anesthetic time of $13 \mathrm{~h} 10 \mathrm{~min}$.

The patient was transferred to the ICU with the endotracheal tube still in place. The patient was sedated using a propofol infusion. After $24 \mathrm{~h}$, the propofol infusion was discontinued, and the patient awoke with a GCS score of (4/E/5) and pupil size of $3 \mathrm{~mm} / 3 \mathrm{~mm}$ (left/right). Afterwards, the sedation agent was switched to fentanyl. After being sedated for $52 \mathrm{~h}$, the patient had awakened with an alert mental status of GCS score of 15 (4/5/6) and no neurologic deficits. Brain CT and MRI scans 3 days after surgery revealed no interval changes. Adequate spontaneous respiration was maintained, and the possibility of brain death was excluded. Extubation was performed on the fourth postoperative day after the patient could communicate. Liver function after transplantation recovered sequentially, and the patient was discharged without any complications.

The presence of a BIS value of zero during surgery is a sign

Corresponding author: Seung Zhoo Yoon, M.D., Department of Anesthesiology and Pain Medicine, College of Medicine, Korea University, 5, Anam-dong, Seongbuk-gu, Seoul 136-705, Korea. Tel: 82-2-920-5632, Fax: 82-2-929-2936, E-mail: yoonsz70@gmail.com

(c) This is an open-access article distributed under the terms of the Creative Commons Attribution Non-Commercial License (http:// creativecommons.org/licenses/by-nc/3.0/), which permits unrestricted non-commercial use, distribution, and reproduction in any medium, provided the original work is properly cited. 
Table 1. Patient Characteristics according to Liver Transplantation Stage

\begin{tabular}{lccc}
\hline & Preanhepatic phase & Anhepatic phase & Neohepatic phase \\
\hline Anesthetic time $(\mathrm{h})$ & 7 & 1 & 5.2 \\
Total fluid $(\mathrm{ml})$ & 12000 & 4000 & 14000 \\
Urine output $(\mathrm{ml})$ & 115 & 0 & 1150 \\
MAP (mmHg) & $70(56-93)$ & $63(56-63)$ & $70(50-93)$ \\
Heart rate $(/ \mathrm{min})$ & $90(85-110)$ & $92(80-105)$ & $93(90-105)$ \\
Body temperature $\left({ }^{\circ} \mathrm{C}\right)$ & $35.4(35.2-35.8)$ & $34.4(34-35)$ & $34.8(34-36)$ \\
Cardiac index $\left(\mathrm{L} / \mathrm{min} / \mathrm{m}^{2}\right)$ & $3.2(2.8-3.4)$ & $3.2(3.0-3.5)$ & $4.2(3.1-5.0)$ \\
pRBC (unit) & 9 & 3 & 10 \\
FFP (unit) & 9 & 3 & 10 \\
Platelet (unit) & & & 20
\end{tabular}

Data expressed as median (range). MAP: mean arterial pressure, pRBC: packed red blood cells, FFP: fresh frozen plasma.

of a poor neurologic prognosis [1]. This case, however, indicates that a BIS value of zero for $10 \mathrm{~h} 50$ min during surgery may not necessarily be correlated with a bad neurological outcome or brain death. The deterioration of consciousness is the most critical complication of fulminant hepatic failure. Therefore, an emergency LT should be performed before irreversible brain damage occurs. Despite active monitoring of brain function in patients undergoing urgent LT, some still suffer brain damage [2]. Therefore, it is important to evaluate a patient's exact brain function status, especially in those with fulminant hepatitis who are already in an unconscious state prior to emergent LT. Brain CT, direct measurement of intracranial pressure, transcranial Doppler imaging, and jugular venous oximetry, can help evaluate cerebral function before surgery [3]. In addition, electroencephalography is the standard method to determine brain function in unconscious patients. One study stated that BIS monitoring may be a useful measurement tool for grading and monitoring the status of hepatic encephalopathy in patients with overt hepatic encephalopathy [4]. Authors reported that mean BIS value decreased in awake patients with chronic liver disease who had more serious conditions $(45.4 \pm 5.0)$ than those with mild conditions $(90.2 \pm 2.5)[4]$.

A BIS value of zero may be due to inadequate anesthesia, hemodynamic instability, hypothermia, insufficient muscle relaxation, artifacts by other people or electric devices, and any major metabolic disturbance. Cerebral hypoperfusion can lead to an acute BIS reduction. However, in our case, hemodynamic variables, including arterial blood pressure and cardiac output, were stable. In addition, we repeatedly rechecked the BIS sensor positioning during the operation and kept the patient's body temperature at $34-35^{\circ} \mathrm{C}$. Given the patient's condition, a relative anesthetic agent overdose cannot completely be excluded. Changes in drug sensitivity of the central nervous system, protein binding, volumes of distribution, drug metabolism, and drug elimination are more common in patients with liver disease [5]. Therefore, an overdose even with the ordinary use of anesthetics may occur in patients with severe liver disease. Fortunately, our patient recovered normally from anesthesia and showed no neurologic deficits after $52 \mathrm{~h}$ postoperatively.

This case has a limitation. We did not monitor BIS during ICU care before and after a BIS value of zero was observed. If we had monitored BIS in the ICU, an in-depth discussion could lead us to a reasonable assumption.

We suggest that a BIS value of zero during surgery is not always correlated with postoperative poor neurological outcome.

\section{References}

1. Misis M, Raxach JG, Molto HP, Vega SM, Rico PS. Bispectral index monitoring for early detection of brain death. Transplant Proc 2008; 40: 1279-81.

2. Kim BS, Lee SG, Hwang S, Park KM, Kim KH, Ahn CS, et al. Neurologic complications in adult living donor liver transplant recipients. Clin Transplant 2007; 21: 544-7.

3. Raghavan M, Marik PE. Therapy of intracranial hypertension in patients with fulminant hepatic failure. Neurocrit Care 2006; 4: $179-89$.

4. Dahaba AA, Worm HC, Zhu SM, Bao FP, Salah A, Zakaria S, et al. Sensitivity and specificity of bispectral index for classification of overt hepatic encephalopathy: a multicentre, observer blinded, validation study. Gut 2008; 57: 77-83.

5. Steadman RH. Anesthesia for liver transplant surgery. Anesthesiol Clin North America 2004; 22: 687-711. 